\title{
Clinical factors and safety of microvascular decompression in the treatment of trigeminal neuralgia.
}

\author{
Chiwen Huang ${ }^{1 \#}$, Zhigang Wan ${ }^{2 \#}$, Caihong Wan ${ }^{3}$, Yunqing $\mathrm{Li}^{4}$, Rongde Zhong,6\#* \\ ${ }^{1}$ Department of Neurosurgery, Ganzhou People's Hospital, Ganzhou, PR China \\ ${ }^{2}$ Department of Neurology, the Second Affiliated Hospital of Nanchang University, Nanchang, PR China \\ ${ }^{3}$ School of Basic Medical Sciences, Capital Medical University, Beijing, PR China \\ ${ }^{4}$ Department of Gastroenterology, Shanghai Tenth People's Hospital, Tongji University, Shanghai, PR China \\ ${ }^{5}$ Medical College of Nanchang University, Nanchang, PR China \\ ${ }^{6}$ Department of Neurosurgery, the Tenth People's Hospital of Shanghai, Shanghai, PR China \\ \#These authors contributed equally
}

\begin{abstract}
Objectives: This study was aimed to investigate the clinical factors and safety of Microvascular Decompression (MVD) in the treatment of Trigeminal Neuralgia (TN).

Methods: The clinical characteristics, intraoperative findings, effectivity, side-effects and score of 215 patients treated with MVD were analysed. The degree of compression of vessels on nerve root was classified into six categories: no contact compression, simple contact, contact and displacement, simple adhesion, adhesion and displacement, and atrophy. Postoperative outcomes included excellent, good, favorable, failure, recurrence, and dead within a follow-up time of 37 months.

Results: Degree of postoperative pain relief were influenced by the factors like: whether symptoms are typical or not, age, duration of disease, whether the results of 3D-tof examination is positive or not, treatment methods before MVD, degree of compression on nerve $(p<0.05$, the correlation was significant), while the gender, the pain location, implicated nerve branch, hypertension, type and quantity of offending vessels were not related to postoperative outcomes. Among them, excellent accounted for $87.9 \%$, good for $4.2 \%$. Patients without pain, complication, nor need of medicine treatment accounted for $74.7 \%$.

Conclusion: Though therapeutic effects of MVD in the treatment of TN is affected by the age of onset, duration of disease and whether symptoms are typical or not, it is surely a safe and effective treatment method.
\end{abstract}

Keywords: Microvascular decompression, Trigeminal neuralgia, Prognosis.

Accepted on March 2, 2018

\section{Introduction}

TN is a recurrent shock-like, knife-cut-like, and tear-like neuropathic pain in the distribution area of cranial nerve $\mathrm{V}$ on the part of face and head, which is considered as one of the most painful clinical diseases. The pain can be triggered by voluntary movements of facial muscles caused by daily activities of talking, smiling, and chewing or by just a random tap on some area of face. Bursts usually last few seconds to tens of seconds and can also even last for several minutes. Patients are normal during remission. With the extension of the course of disease, the attack becomes more frequent with a shorter interval, and the pain get intensified, which will seriously affect the life quality of patients, even lead to a mental disorder or a suicide [1]. Fortunately, TN is rare with an incidence of 8/10000 years. Among 10000 people, lifetime prevalence only 0.7 person. However, this number might have been underestimated, for that many cases have been misdiagnosed with dental problems at the beginning and received many unnecessary treatments [2]. According to etiologic classification of Giorgio Cruccu, TN can be divided into idiopathic TN, typical TN, and secondary TN [3]. At early stage, medicines like carbamazepine or oxcarbazepine and other psychotropic drugs were used to control or relief pain for TN patients, while there are about $30-75 \%$ patients had unsatisfactory therapeutic pain-relief effects or side effects after a long-term medication, thus they had to choose surgery 
which have a high surgical risk [4,5]. Till now, there is still not a uniform mechanism clarifying pathogenesis of TN. But most scholars believed in "compression-short circuit theory": Area of trigeminal nerve entering pons is a naked area of about several millimeters long and without myelin sheath surrounded, as a transitional zone around the central nerve and peripheral nerve (root entry zone, REZ), which is affected by pulsating blood vessels and others, compressed trigeminal will lead to demyelination, when naked axons close to each other, resulting in short-circuits not only between pain fibers and non-pain fibers, but also between afferent fibers and efferent fibers, thus spontaneous and ectopic nervous impulses (like tenderness) were transmitted through false synapses, and recognized as algesia leading to TN [6-9]. MVD is an treatment method of TN through separating compressed nerve and offending vessel and filling Teflon between them, which has become the first choice for functional neurosurgery in treating primary $\mathrm{TN}$, because of its significant therapeutic effect, safety, low recurrent rate, and preservation of nervous and vascular functions [10,11]. However, many studies reported that not all TN patients after MVD can get pain controlled, and some patients only got pain relief or even no effect while with risks of recrudescence and complications $[12,13]$. So, questions: 1 . What is the factors that influence the postoperative effects of MVD in the treatment of TN; 2 . How is the validity and safety of MVD in the treatment of TN? There were a few studies focused on these studies, but it is limited with small sample sizes and short-term follow-ups. Besides, there were no assessment of MVD considering both therapeutic effects and complications. This study aims to solve above mentioned problems, to explore clinical significance of MVD in the treatment of TN (excluding secondary TN).

\section{Materials and Methods}

\section{Patients and eligibility criteria}

Exclusion criteria were as follows: patients with $\mathrm{TN}$ caused by multiple sclerosis, tumor, arterial aneurysm and so on, patients cannot tolerate general anesthesia and/or surgery, patients with contraindication of Magnetic Resonance Imaging (MRI), patients refusing follow-up, patients with non-death interrupted follow-up.

Diagnosis of TN, including both typical TN and atypical TN, was made by a team of functional neurosurgeons according to the diagnostic criteria (International classification of headache disorders-3) established by International Headache Society.

From July 2008 to June 2013, 215 TN patients received MVD in Department of Neurosurgery, Shanghai Tenth People's Hospital. Clinical data were recorded and analysed as following: (1) gender, age, symptoms (typical and atypical), and location of pain; (2) impacted nerve branches, duration of disease, history of hypertension; (3) 3D-tof test results: positive, negative, or probable positive (two imaging specialists and one functional neurosurgeon professor film reading separately); (4) treatments received before MVD, including medicine, MVD, and others (gamma knife, nerve block, physical therapy, radiofrequency thermocoagulation and so on); (5) offending vessel and compression degree of trigeminal: no contact compression, simple contact, contact and displacement, simple adhesion, adhesion and displacement, and atrophy, which was determined by the surgeon and another neurosurgeon who watched the surgical video together; (6) 37-month-long follow-ups for therapeutic effects and complications by telephone, messages, emails and clinics, and therapeutic effects were classified into: 1. excellent (no pain and no medicine needed after surgery); 2. Good (pain relief $>50 \%$, no or occasional medicine needed); 3. Favorable (pain relief or decreased medicine $\geq 50 \%$, and multiple branch pain degraded to simple branch pain); 4. failure (no pain relief); 5. Recurrence (recurrent pain after disappeared pain or after a short-term pain relief); 6. dead; (7) score (effects and complications): 0 point (excellent and no complication); 1 point (excellent and neurosis negative), 2 points (excellent and neurosis positive), 3 points (good and no complication), 4 points (good and neurosis negative), 5 points (good and neurosis positive); 6 points (favorable and no complication), 7 points (favorable and neurosis negative), 8 points (favorable and neurosis positive); 9 points (failure and no complication), 10 points (failure and neurosis negative), 11 points (failure and neurosis positive); 12 points (recurrence); and 13 points (dead).

\section{Surgical procedure}

Surgeries were all operated by senior functional neurosurgeons. After general anesthesia, endotracheal intubation was done. Patients were laid in a lateral position with unaffected side downward, and head was $15^{\circ}$ prolapse and rotated $10^{\circ}$ toward the unaffected side, neck was slightly anteflexed, allowing 2-finger-breadth between mandible and sternum, and with mastoid process in the highest position. A transverse incision of $3-5 \mathrm{~cm}$ were made where $1 \mathrm{~cm}$ over the flexure of occipital bone towards basis cranii within the hairline behind ear, which was convenient for operations under microscope. Then, bone flap of $2.0 \mathrm{~cm} \times 2.5 \mathrm{~cm}$ was removed, with upper edge exposed to transverse sinus, lateral to the posterior margin of the sinuses sigmoideus, the intersection of transverse sinus and sigmoid sinus was exposed. Dural was curved-cut and overhung. Under microscope, cerebrospinal fluid in cisterna magna and lateral cisterna magna was released first. After cerebellum was sunken, arachnoid membrane at cerebellopontine angle was dissected with sharp dissection. Then, trigeminal was exposed and wholly explored from Meckel capsule to roots of trigeminal nerve at pons, find out the origin, category, number of compression vessels, and location and degree of compressed nerves. Teflon was placed between offending vessels and brainstem keeping offending vessels away from REZ. If no certain vessels were found, corresponding nerves should also be teased, and partial neurotomy of the most possible nerve fiber bundle should be conducted. Then, hemostasis was performed in surgical filed, cerebral dura mater was perfectly sutured, and biogel was adopted for stickup. Drainage tube was not needed. 


\section{Statistical analysis}

SPSS23.0 software was used for statistical analysis. Rank correlation was used to analyse the relationship between two variables. Frequency was used to describe the frequency of appearance and percentage of sub-variables.

\section{Informed consent and ethics statement}

Every patient had read and signed the operation informed consent. The research protocol approved by the ethics committee to sign.

\section{Results}

There were 215 patients who received MVD and been followed up for 37 months after surgeries, of which $41.4 \%$ were male, and $58.6 \%$ were females. Typical $\mathrm{TN}$ accounted for $83.1 \%$, and atypical TN accounted for $14.9 \%$. The average age was $62.9 \mathrm{y}$ ranging from 23 to $85 \mathrm{y}$. The average age of typical TN (61.8 y) was smaller than atypical TN (69.0 y). The duration of disease last from 1 month to $40 \mathrm{y}$, with an average of 5.9 y. $44.7 \%$ of the patients had duration of disease less than three years. $127(59.1 \%)$ patients had pain on the right side, 87 $(40.5 \%)$ on the left side, and only $1(0.4 \%)$ patient on both sides, non-attacked at the same time and the right was more frequent. The number of patients who had sole pain at the first, second and third branch of trigeminal nerve was 3.3\%, 20.5\% and $4018.6 \%$ respectively. However, pain involved the second and third branch simultaneously was more common, taking up to $33.5 \%$ cases. $3516.3 \%$ patients had pain at both the first and second branch. $7.9 \%$ patients had pain on a whole side face (involving three branches simultaneously). 74 (34.4\%) cases have a history of hypertension. Treatments before MVD were as follows: $91(42.3 \%)$ cases had medicines, 23 (10.7\%) cases received MVD, $59(27.4 \%)$ cases had other treatments, and 30 $(14.0 \%)$ cases had multiple treatments, only $12(5.6 \%)$ patients with short duration of disease received no treatment. 3D-tof test showed that $191(88.8 \%)$ cases were positive, $9(4.2 \%)$ negative, and $15(7.0 \%)$ probable positive. Except for $6(2.8 \%)$ cases, there were offending vessel compressing trigeminal detected during surgeries. There were Vertebral Artery (VA) that compression found in $26(12.1 \%)$ cases, Superior Cerebellar Artery (SCA) in $73(34.0 \%)$ cases, Anterior Inferior Cerebellar Artery (AICA) in $25(11.6 \%)$ cases, Posterior Inferior Cerebellar Artery (PICA) in 12 (5.6\%) cases, multiple arteries in $37(17.2 \%)$ cases, vein compression in $4(1.9 \%)$ cases, arteriovenous compression in $24(11.2 \%)$ cases, multiple veins compression in $4(1.9 \%)$ cases, and no vessel types can be identified during operation or through surgery video in 4 (1.9\%) cases. During operations, between the vessel and nerve, simple contact was found in $46(21.4 \%)$ cases, contact and displacement in $22(10.2 \%)$ cases, simple adhesion in 57
(26.5\%) cases, adhesion and displacement in 64 (29.8\%) cases, and nerve atrophy in $20(9.3 \%)$ cases. Age was positively correlated with compression degree of nerves (Table 1). As shown in Table 1, degree of postoperative pain relief was influenced by whether symptoms are typical or not, age of onset, duration of disease, 3D-tof test results, treatments before MVD, degree of compression on nerves $(p<0.05)$, while gender, pain location, implicated nerve branches, hypertension, type of offending vessels, and number of offending vessels were not related to postoperative therapeutic effects.

Follow-up lasted for 37 months. The results were excellent for $189(87.9 \%)$ cases, good for 9 cases, favorable for 4 cases, failure for 4 cases, recurrence for 7 cases, and dead for 2 cases, of which 1 patient died of cerebellar infarction hemorrhage seven days after operation, and the other patient died of acute heart failure. Almost all patients suffered from transient facial numbness, and in $18(8.4 \%)$ cases were remaining numbness after 37 months. There was facial paralysis in 4 cases, hearing loss in the same side in 3 cases, cerebellar injury in 11 cases. 8 patients' discharge was delayed: 5 patients needed antibiotics due to infection of incisional wound after surgeries and 3 patients needed dural repair with surgery due to leakage of cerebrospinal fluid (Table 2), all of whom recovered with different degree within two weeks after surgeries. As the total score (for therapeutic effects and complication) shown in Figure 1, yellow indicates 0 point, accounting for $74.4 \%$. It meant no pain and complication after surgeries, suggesting no medicine treatment needed. 16 cases got 1 point. 7 cases had recurrent pain ( 1 case within 1 year, 2 cases within 2 years, and 4 cases within 3 years).

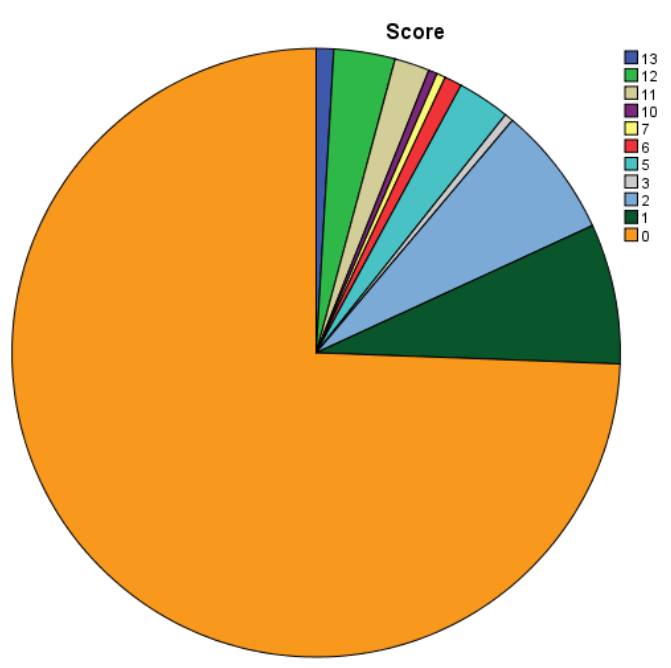

Figure 1. Pie chart of scores after operations (therapeutic effects +complications).

Table 1. Frequency distribution and correlation analysis of clinical data before and after operations.

\begin{tabular}{lll}
\hline Relevant factors & Frequency & Percentage (\%) \\
\hline
\end{tabular}




\begin{tabular}{|c|c|c|c|c|c|}
\hline \multirow[t]{2}{*}{ Sex } & $\mathrm{F}$ & 126 & 58.6 & 0.004 & 0.958 \\
\hline & $M$ & 89 & 41.4 & & \\
\hline \multirow[t]{2}{*}{ Symptom } & Typical & 183 & 85.1 & $0.518^{* *}$ & 0.0001 \\
\hline & Atypical & 32 & 14.9 & & \\
\hline \multirow[t]{3}{*}{$\operatorname{Age}(y)$} & $\leq 50$ & 31 & 14.4 & $0.140^{*}$ & 0.041 \\
\hline & $51-70$ & 122 & 56.7 & & \\
\hline & $>70$ & 62 & 28.8 & & \\
\hline \multirow[t]{6}{*}{ Branch } & $\mathrm{v} 1$ & 7 & 3.3 & 0.07 & 0.307 \\
\hline & v2 & 44 & 20.5 & & \\
\hline & v3 & 40 & 18.6 & & \\
\hline & $\mathrm{v} 1+\mathrm{v} 2$ & 35 & 16.3 & & \\
\hline & $\mathrm{v} 2+\mathrm{v} 3$ & 72 & 33.5 & & \\
\hline & $\mathrm{v} 1+\mathrm{v} 2+\mathrm{v} 3$ & 17 & 7.9 & & \\
\hline \multirow[t]{3}{*}{ Side } & $\mathrm{L}$ & 87 & 40.5 & -0.078 & 0.252 \\
\hline & $\mathrm{R}$ & 127 & 59.1 & & \\
\hline & $L+R$ & 1 & 0.5 & & \\
\hline \multirow[t]{4}{*}{ Duration } & $\leq 3 Y$ & 96 & 44.7 & $0.491^{* *}$ & 0.0003 \\
\hline & $4-6 Y$ & 55 & 25.6 & & \\
\hline & $7-10 Y$ & 26 & 12.1 & & \\
\hline & $>10 \mathrm{Y}$ & 38 & 17.7 & & \\
\hline \multirow[t]{3}{*}{ 3D-tof } & Positive & 191 & 88.8 & $0.184^{* *}$ & 0.007 \\
\hline & Negative & 9 & 4.2 & & \\
\hline & Uncertainty & 15 & 7 & & \\
\hline \multirow[t]{2}{*}{ Hypertension } & Positive & 74 & 34.4 & 0.06 & 0.382 \\
\hline & Negative & 141 & 65.6 & & \\
\hline \multirow[t]{6}{*}{ History of treatments } & No treatment & 12 & 5.6 & $0.161^{*}$ & 0.018 \\
\hline & MVD & 23 & 10.7 & & \\
\hline & Medication & 91 & 42.3 & & \\
\hline & Others treatments & 59 & 27.4 & & \\
\hline & Multiple treatments & 30 & 14 & & \\
\hline & No finging & 6 & 2.8 & & \\
\hline \multirow[t]{7}{*}{ Vessel } & VA & 26 & 12.1 & 0.053 & 0.443 \\
\hline & SCA & 73 & 34 & & \\
\hline & AICA & 25 & 11.6 & & \\
\hline & PICA & 12 & 5.6 & & \\
\hline & Unnamed vessel & 4 & 1.9 & & \\
\hline & Vein & 4 & 1.9 & & \\
\hline & Artery+Vein & 24 & 11.2 & & \\
\hline
\end{tabular}




\begin{tabular}{llll}
\cline { 2 - 3 } Multiple artery & 37 & 17.2 \\
\cline { 2 - 4 } Multiple vein & 4 & 1.9 \\
$0.436^{* *}$ & 2.0 \\
\hline No compressing & 6 & 21.4 \\
\cline { 1 - 3 } Contact & 46 & 10.2 \\
\hline Contact and indentation & 22 & 26.5 \\
\hline Adhesion & 57 & 29.8 \\
\hline Adhesion and indentation & 64 & 9.3
\end{tabular}

*At the level of 0.05 (double tail), the correlation was significant. *At the level of 0.01 (double tail), the correlation was significant. VA: Vertebral Artery; SCA: Arteriae Cerebelli Superior; AICA: Anterior Inferior Cerebellar Artery; PICA: Posterior Inferior Cerebellar Artery.

Table 2. Frequency distribution of therapeutic effects and complications for follow-ups after operations.

\begin{tabular}{llll}
\hline Variables & & Frequency & Percentage (\%) \\
\hline Outcome & Excellent & 189 & 87.9 \\
\cline { 2 - 4 } & Good & 9 & 4.2 \\
\cline { 2 - 4 } & Recurrence & 7 & 3.3 \\
\cline { 2 - 4 } & Failure & 4 & 1.9 \\
\cline { 2 - 4 } & Favorable & 4 & 1.9 \\
\cline { 2 - 4 } & Dead & 2 & 0.9 \\
\cline { 2 - 4 } & Normal & 162 & 75.3 \\
\hline Complication & Facial numbness & 18 & 8.4 \\
\cline { 2 - 4 } & Cerebellar injury symptoms & 11 & 5.1 \\
\cline { 2 - 4 } & Recurrence & 7 & 3.3 \\
\cline { 2 - 4 } & Infection & 5 & 2.3 \\
\cline { 2 - 4 } & Facial palsy & 4 & 1.9 \\
\cline { 2 - 4 } & CSF leak & 3 & 1.4 \\
\cline { 2 - 4 } & Hearing deficit & 2 & 0.9 \\
\cline { 2 - 4 } & Dead & 3 & \\
\hline
\end{tabular}

\section{Discussion}

TN was described as an unforgettable malignant progressive disease, because of its shock-like and tear-like neuropathic pain in the face, and during the course of disease, the attack becomes more frequent, interval becomes shorter, and the pain gets severer. $87 \%$ patients were left horrible memories due to the first onset server pain, and only $13 \%$ patients had continuous dull pain for the first time. They may refuse to talk, eat, brush teeth or smile due to fear of pain [14]. Many publications reported that females had higher risks of TN than males. In our retrospective report, the male/female ratio of the patients was 0.71 . Joanna believed that $\mathrm{TN}$ was related with hypertension, especially for females, however, only $34.4 \%$ of 215 patients had a history of hypertension, and she also found that few patients had migraine or chronic pain, which was consistent with our retrospective study [2]. Also, a few patients showed autonomic symptoms during bedside physical examination among these 215 patients, lower than the results of $31 \%[15,16]$ described by Stine et al. might due to improved symptoms during bedside physical examination. According to lasing time of pain and whether or not dull pain at interval, TN can be classified as typical TN or atypical TN. Atypical TN was defined as continuous pain or appearance of dull pain at interval, with or without paresthesia or autonomic symptoms, and laboratory tests and imaging were usually normal. Atypical $\mathrm{TN}$ is a result of long-term onsets of typical TN, while Stine suggested that typical TN might suffer from atypical TN before. Atypical TN was more common in females than males, which might be related to longer lifetime in females, but still needs further studying. Among these 215 cases, 32 cases were atypical TN with a higher age than typical TN (68.0 y vs. 61.8 y), 10 cases were females, and almost all nerves were compressed to a significant degree. Stine believed that atypical TN was more progressive and had poorer therapeutic effects than typical TN, which was consistent with the results in Table 1 , might due to long curse of disease, low positive rate of 3Dtof test, and obvious compression on nerve in atypical TN [15]. 3D-tof (Magnetic Resonance Angiography) MRA, which had significance in excluding malignancies, and clarifying relationship between offending vessels and trigeminal, and degree of compression, consistent with observation during surgeries, was the first imaging test method of diagnosing TN, and helpful for MVD $[17,18]$. Table 1 indicated that positive rate in $3 \mathrm{D}$-tod MRA was $88.8 \%$, probable positive rate $7.0 \%$, and negative rate $4.2 \%$. Probable positive or negative results were related to low flow related enhancement. During surgeries or surgical videos, probable positive and negative results were common in patients with vein compression and server compression on nerves. 3D-tof MRA, together with 3D-fiesta MRA and DTL, can increase the specificity and sensitivity in diagnosing TN significantly, confirm compression degree of nerves, and provide useful prediction for MVD.

Psychotropic drugs like carbamazepine, olanzapine and lamotrigine can relief pain at early stage of disease, but there may be side effects like somnolence, leukopenia, liver injury and ataxia, and possibility of loss of efficacy after long-term 
usage, the failure rate was more than 50\%. Radiofrequency thermocoagulation, glycerol gangliolysis, and balloon tamponade were minimal-invasive in treating $\mathrm{TN}$, with a low disability rate and lethality, which destroy nerve root leading to sensory deprivation and keratitis, had a high recurrent rate, and cannot relief pain timely $[1,2,19]$. MVD hadobvious advantages compared with them. MVD is to an etiologic treatment of TN through separating compressed nerve and offending vessel and filling Teflon instead, with significant therapeutic effect, safety, low recurrent rate, and preservation of nervous and vascular functions [10,11]. Questionnaire survey respondents (TN patients treated with MVD) indicated that most of them were satisfactory with the surgeries, and they wanted to have surgical treatment at early stage of disease [20]. Pollock BE reported that $70-80 \%$ patients got complete remission of pain after $\mathrm{MVD}$, and the pain relief rate was as high as of $60-70 \%$ even 10-20 y after surgeries [21,22]. According to the study of Barker FG 2nd, 70\% patients got excellent 10 y after MVD, $2 \%$ patients got recurrence within 5 years, $1 \%$ got recurrence within $10 \mathrm{y}$ [23]. As shown in Table $2,87.9 \%$ cases get excellent result, $4.2 \%$ good. Patients with 0 point are accounting for $74.4 \%$, who had no pain or complication after the surgeries, suggesting no medicine treatment needed (Figure 1). Though with senior surgeons, there were still risks with MVD, due to craniotomy and general anesthesia. Main influences of MVD were facial numbness and cerebellar injury sign, while the two cases with postoperative complications both got improved after surgeries in this study. 3 (1.4\%) cases had hearing loss, which was lower than $5 \%$ reported by Zakrzewska [2], he believed that the hearing loss was permanent, and hearing recovery has not been found in this study either. Other complications included facioplegia, infection of incisional wound, and leakage of cerebrospinal fluid. According to systemic retrospective analysis, TN patients could get good outcomes if receiving surgery of MVD with four years after diagnosis. From the pie of scores after operations, it was easily to find that MVD had a high safety in treating TN. And the area of yellow was larger than the total area of the other colors ( 1 point in $16(7.4 \%)$ cases $>$ two points in $15(7.0 \%)$ cases $>$ five points in six $(2.8 \%)$ cases $)$. Two $(0.9 \%)$ patients died: one patient died of cerebellar infarction hemorrhage, and the other one patient died of acute heart failure (Figure 1).

In order to find out what factors can influence the therapeutic effects of MVD on TN, we did rank correlation analysis. Results showed that the therapeutic effects were closely related to following factors: 1 . How long was course of disease? Only 1 patient had favorable result and 1 patient got failure result among 96 cases with a course of disease less than 3 years, expect for dead or recurrence, while 4 patients got favorable result, 4 patients got failure result, and 5 patients got recurrence result among 38 cases with a course of disease $>10$ y. Thus, shorter course of disease indicated better therapeutic effects, and vice versa. 2. Were symptoms before surgeries typical or not? The case number of favorable, failure and recurrence were 1, 0, 3 in typical $\mathrm{TN}$, and 3, 4, 4 in atypical $\mathrm{TN}$ respectively, indicating that compression on nerves got severer, typical TN transferred into atypical TN, and surgical effects got worse, as course of disease prolonged. It was great to perform MVD when symptoms were typical, in order to achieve satisfactory therapeutic effects. 3. The degree of compression of vessels to the trigeminal nerve, correlation analysis showed statistical significance $(\mathrm{P}<0.05)$, and correlation coefficient was 0.436 , suggesting that the less compression, the easier the surgery, and better therapeutic effects of MVD. Besides, probable positive or negative result of 3D-tof test, and multiple treatments before MVD were not beneficial to pain relief $(\mathrm{P}<0.05)$. And, older age could reduce possibility of pain relief. Gender, pain location, involved nerve branch, hypertension, and offending vessel type and number were not related to therapeutic effects (Table 1). Some scholars believed that compressed vessel type and number were related to prognosis, while rank correlation analysis showed no significance in this study. However, pain emission rate and recurrence rate in vein compression group and arteriovenous compression group was significantly different from those in artery compression group. It was unable to conduct multifactor logistic regression and kappa analysis to clarify the exact relationship between sub- variables and therapeutic effects, due to small sample size in sub-variables, and missed levels or grades. There were still limitations in evaluating factors influencing therapeutic effects of MVD on TN and its safety in this study, like that there was no variable describing pain attack times and interval time, follow-ups were not divided into periods, and there were no other treatments like radiofrequency thermocoagulation as controls.

In summary, therapeutic effects of MVD in the treatment of TN can be influenced by many factors. It is necessary to conduct a detailed analysis of patient's clinical characteristics, and to predict and to tell the surgical therapeutic effects to patient. Generally speaking, MVD is an effective, safe and permanent therapeutic method for the treatment of $\mathrm{TN}$, while radiofrequency thermocoagulation is also a good choice for patients like the elder who cannot tolerate MVD.

\section{References}

1. Zakrzewska JM, Linskey ME. Trigeminal neuralgia. BMJ 2015; 350: 1238.

2. Zakrzewska JM, Linskey ME. Trigeminal neuralgia. BMJ Clin Evid 2014; 2014.

3. Cruccu G, Finnerup NB, Jensen TS. Trigeminal neuralgia: New classification and diagnostic grading for practice and research. Neurology 2016; 87: 220-228.

4. Jensen TS. Selective sodium channel blockers in trigeminal neuralgia. Lancet Neurol 2017; 16: 255-256.

5. Zakrzewska JM, Palmer J, Morisset V. Safety and efficacy of a Nav1.7 selective sodium channel blocker in patients with trigeminal neuralgia: a double-blind, placebocontrolled, randomised withdrawal phase 2a trial. Lancet Neurol 2017; 16: 291-300.

6. Love S, Gradidge T, Coakham HB. Trigeminal neuralgia due to multiple sclerosis: ultrastructural findings in 
trigeminal rhizotomy specimens. Neuropathol Appl Neurobiol 2001; 27: 238-244.

7. Smith KJ, Mcdonald WI. Spontaneous and evoked electrical discharges from a central demyelinating lesion. J Neurol Sci 1982; 55: 39-47.

8. Devor M, Amir R, Rappaport ZH. Pathophysiology of trigeminal neuralgia: the ignition Hypothesis. Clin J Pain 2002; 18: 4-13.

9. Hilton DA, Love S, Gradidge T. Pathological findings associated with trigeminal neuralgia caused by vascular compression. Neurosurgery 1994; 35: 299-303.

10. Ibrahim S. Trigeminal neuralgia: diagnostic criteria, clinical aspects and treatment outcomes. A retrospective study. Gerodontology 2014; 31: 89-94.

11. Ko AL, Ozpinar A, Lee A. Long-term efficacy and safety of internal neurolysis for trigeminal neuralgia without neurovascular compression. J Neurosurg 2015; 122: 1048-1057.

12. Merrison AF, Fuller G. Treatment options for trigeminal neuralgia. BMJ 2003; 327: 1360-1361.

13. Gerganov VM, Giordano M, Elolf E. Operative management of patients with radiosurgery-related trigeminal neuralgia: analysis of the surgical morbidity and pain outcome. Clin Neurol Neurosurg 2014; 122: 23-28.

14. Xia L, Zhong J, Zhu J. Effectiveness and safety of microvascular decompression surgery for treatment of trigeminal neuralgia: a systematic review. J Craniofac Surg 2014; 25: 1413-1417.

15. Maarbjerg S, Wolfram F, Gozalov A. Significance of neurovascular contact in classical trigeminal neuralgia. Brain 2015; 138: 311-319.

16. Weiss AL, Ehrhardt KP, Tolba R. Atypical facial pain: a comprehensive, evidence-based review. Curr Pain Headache Rep 2017; 21: 8 .
17. Desouza DD, Hodaie M, Davis KD. Abnormal trigeminal nerve microstructure and brain white matter in idiopathic trigeminal neuralgia. Pain 2014; 155: 37-44.

18. Antonini G, Di Pasquale A, Cruccu G. Magnetic resonance imaging contribution for diagnosing symptomatic neurovascular contact in classical trigeminal neuralgia: a blinded case-control study and meta-analysis. Pain 2014; 155: 1464-1471.

19. Brisman R. Gamma knife radiosurgery for primary management for trigeminal neuralgia. J Neurosurg; 2000; 93: 159-161.

20. Yamahata H, Tokimura H, Hanaya R. Microvascular decompression for trigeminal neuralgia due to compression by the vertebral artery: report of 3 cases. Minim Invasive Neurosurg 2011; 54: 79-82.

21. Pollock BE. Surgical management of medically refractory trigeminal neuralgia. Curr Neurol Neurosci Rep 2012; 12: 125-131.

22. Pollock BE, Phuong LK, Gorman DA. Stereotactic radiosurgery for idiopathic trigeminal neuralgia. J Neurosurg 2002; 97: 347-353.

23. Barker FN, Jannetta PJ, Bissonette DJ. The long-term outcome of microvascular decompression for trigeminal neuralgia. N Engl J Med 1996; 334: 1077-1083.

\section{*Correspondence to}

Rongde Zhong

Department of Neurosurgery

The Tenth People's Hospital of Shanghai

PR China 